UFIFT-HEP-05-6

MCTP-05-65

\title{
Cabibbo Haze in Lepton Mixing
}

\author{
AseshKrishna Datta ${ }^{1,2}$, Lisa Everett ${ }^{1}$, and Pierre Ramond ${ }^{1}$ \\ 1. Institute for Fundamental Theory, Department of Physics \\ University of Florida, Gainesville, FL, 32611, USA \\ 2. Michigan Center for Theoretical Physics, Department of Physics \\ University of Michigan, Ann Arbor, MI, 48109, USA
}

\begin{abstract}
Quark-lepton unification suggests Cabibbo-sized effects in lepton mixings, which we call Cabibbo haze. We give simple examples and explore possible Wolfenstein-like parametrizations of the MNSP matrix which incorporate leptonic Cabibbo shifts. We find that the size of the $\mathrm{CHOOZ}$ angle is not always correlated with the observability of $\mathrm{CP}$ violation.
\end{abstract}




\section{Introduction}

With recent experimental progress in the neutrino sector, a quantitative picture of the Maki-Nakagawa-SakataPontecorvo (MNSP) lepton mixing matrix [1, 2] has begun to emerge. In contrast to the Cabibbo-KobayashiMaskawa (CKM) quark mixing matrix, which is approximately the identity matrix up to effects of order the Cabibbo angle $\lambda \equiv \sin \theta_{c} \sim 0.22$, the MNSP matrix contains two large angles $\theta_{\odot}, \theta_{\oplus}$, and one small angle $\theta_{13}$ :

$$
\mathcal{U}_{M N S P} \simeq\left(\begin{array}{ccc}
\cos \theta_{\odot} & \sin \theta_{\odot} & \sin \theta_{13} \\
-\cos \theta_{\oplus} \sin \theta_{\odot} & \cos \theta_{\oplus} \cos \theta_{\odot} & \sin \theta_{\oplus} \\
\sin \theta_{\oplus} \sin \theta_{\odot} & -\sin \theta_{\oplus} \cos \theta_{\odot} & \cos \theta_{\oplus}
\end{array}\right),
$$

for which combined data from the solar (with SNO-salt [3] and Super-Kamiokande (SK) [4]), atmospheric (from the latest SK data [5]), reactor (KamLAND [6], CHOOZ and Palo Verde [7]), and accelerator experiments $(\mathrm{K} 2 \mathrm{~K})\left[8\right.$ indicates at $3 \sigma$ the following constraints on the atmospheric and $\mathrm{CHOOZ}$ angles $[9]{ }^{1}$

$$
\theta_{\oplus}=45_{-10^{\circ}}^{+10^{\circ}} ; \quad \theta_{13}<13^{\circ},
$$

while the solar angle is constrained to a rather precise range of

$$
\theta_{\odot}=32.5_{-2.3^{\circ}}^{\circ}
$$

as indicated by the SNO-salt data when combined with the data from the KamLAND reactor experiment. Note that the limiting value of $\theta_{13}$ is approximately equal to $\theta_{c}$.

Understanding the origin of the MNSP mixing angles provides a new perspective and challenges for addressing the flavor puzzles of the Standard Model (SM). In this letter, we work in the context of quark-lepton grand unification [12, for which all available quark and lepton sector data can be synthesized in the quest toward a credible theory of flavor. The qualitative differences between the quark and lepton mixing matrices, while perhaps surprising, are not inconsistent with grand unification. The seesaw mechanism [13 introduces a new unitary matrix into the MNSP matrix, with no analogue in the quark sector [14, that can provide a source for the discrepancy. Explaining the observed pattern of two large and one small lepton mixing angles without fine-tuning in a three-family model is the Achilles' heel for most model-building attempts.

Wolfenstein's parametrization of the CKM matrix [15] as an expansion in powers of $\lambda$ not only has wellknown practical advantages for phenomenology, but also provides a theoretical framework for examining the quark sector in the $\lambda \rightarrow 0$ limit. One aim of this letter is to seek a similar parametrization of the MNSP matrix. We begin by demonstrating that grand unification implies that Cabibbo-sized effects are expected in the lepton as well as the quark sector. Such effects create a Cabibbo haze in lepton flavor mixing, keeping in mind that unlike the CKM matrix, the MNSP matrix is not known in the $\lambda \rightarrow 0$ limit, except that it contains two large angles. Leptonic Cabibbo-sized perturbations can then shift the atmospheric and solar angles from their unknown initial values by $\sim \theta_{c}$, provide the dominant source for the $\mathrm{CHOOZ}$ angle, and dictate the size of CP-violating effects. Although the data is not yet precise enough to pinpoint a particular Wolfenstein-like parametrization, regularities may emerge upon more precise measurements of the MNSP parameters.

\section{Seesaw and Grand Unification}

In the SM, $\Delta I_{\mathrm{w}}=1 / 2$ electroweak breaking generates Dirac masses for the quarks and charged leptons through Yukawa couplings. These Dirac mass matrices are diagonalized by biunitary transformations, in which $\mathcal{U}_{2 / 3}$, $\mathcal{U}_{-1 / 3}$, and $\mathcal{U}_{-1}$ are the rotation matrices for the left-handed states, while $\mathcal{V}_{2 / 3}, \mathcal{V}_{-1 / 3}$, and $\mathcal{V}_{-1}$ are the rotation matrices for the right-handed states. Physical observables depend only on the CKM matrix

$$
\mathcal{U}_{C K M}=\mathcal{U}_{2 / 3}^{\dagger} \mathcal{U}_{-1 / 3}
$$

In the original formulation of the SM, all three neutrinos are massless and lepton mixing is unobservable. Nonvanishing neutrino masses require one to go beyond the SM, and add e.g. one right-handed neutrino for each

\footnotetext{
${ }^{1}$ Significant improvements in constraining $\theta_{13}$ (down to $3^{\circ}$ or below) are foreseen at the planned reactor neutrino experiments, superbeams and/or neutrino factories [10; not much improvement in the atmospheric sector is foreseen [1].
} 
family. Electroweak breaking then also generates Dirac mass terms for the neutrinos, with $\mathcal{M}_{\text {Dirac }}^{(0)}=\mathcal{U}_{0} \mathcal{D}_{0} \mathcal{V}_{0}^{\dagger}$. If there is no seesaw, the Dirac mass eigenvalues $m_{i}^{\mathrm{D}}\left(\mathcal{D}_{0}=\operatorname{diag}\left(m_{i}^{\mathrm{D}}\right)\right)$ are the physical neutrino masses. However, large $\Delta I_{\mathrm{w}}=0$ mass terms for the right-handed neutrinos arise naturally, as they are unsuppressed by gauge quantum numbers. This Majorana mass matrix $\mathcal{M}_{M a j}^{(0)}$ has entries which can be much larger than the electroweak scale. After the seesaw,

$$
\mathcal{M}_{\text {Seesaw }}^{(0)}=\mathcal{M}_{\text {Dirac }}^{(0)} \frac{1}{\mathcal{M}_{\text {Maj }}^{(0)}} \mathcal{M}_{\text {Dirac }}^{(0) T}
$$

which can be reexpressed [14] as

$$
\begin{aligned}
\mathcal{M}_{\text {Seesaw }}^{(0)} & =\mathcal{U}_{0} \mathcal{D}_{0} \mathcal{V}_{0}^{\dagger} \frac{1}{\mathcal{M}_{M a j}^{(0)}} \mathcal{V}_{0}^{*} \mathcal{D}_{0} \mathcal{U}_{0}^{T} \\
& =\mathcal{U}_{0} \mathcal{C} \mathcal{U}_{0}^{T}
\end{aligned}
$$

$\mathcal{C}$ is the central matrix

$$
\mathcal{C} \equiv \mathcal{D}_{0} \mathcal{V}_{0}^{\dagger} \frac{1}{\mathcal{M}_{M a j}^{(0)}} \mathcal{V}_{0}^{*} \mathcal{D}_{0}
$$

diagonalized by the unitary matrix $\mathcal{F}$

$$
\mathcal{C}=\mathcal{F} \mathcal{D}_{\nu} \mathcal{F}^{T}
$$

where $\mathcal{D}_{\nu}$ is the diagonal matrix of the physical neutrino masses $m_{1}, m_{2}$, and $m_{3}$. The MNSP matrix can then immediately be written in the suggestive form

$$
\mathcal{U}_{M N S P}=\mathcal{U}_{-1}^{\dagger} \mathcal{U}_{0} \mathcal{F}
$$

Eq. (9) highlights the differences between $\mathcal{U}_{M N S P}$ and $\mathcal{U}_{C K M}$, and provides the basis for our discussion.

Grand unification suggests connections between the MNSP and CKM matrices. The simplest Higgs structures lead to the following relations

$$
\begin{array}{rlrl}
S U(5): & : & & \mathcal{M}^{(-1 / 3)} \\
& \sim \mathcal{M}^{(-1) T} . \\
S O(10): & & \mathcal{M}^{(2 / 3)} \sim \mathcal{M}_{\text {Dirac }}^{(0)}
\end{array}
$$

which imply

so that

$$
\mathcal{U}_{-1 / 3} \sim \mathcal{V}_{-1}^{*} ; \quad \mathcal{U}_{2 / 3} \sim \mathcal{U}_{0}
$$

$$
\mathcal{U}_{M N S P}=\mathcal{V}_{-1 / 3}^{T} \mathcal{U}_{-1 / 3} \mathcal{U}_{C K M}^{\dagger} \mathcal{F}
$$

Models can then be categorized according to the structure of the charge $-1 / 3$ Yukawa couplings and the number of large angles in $\mathcal{F}$. A particularly illustrative example [16] is the class of models with symmetric $\mathcal{M}_{-1 / 3}$, for which

$$
\mathcal{U}_{-1 / 3}=\mathcal{V}_{-1 / 3}^{*}
$$

which implies that the MNSP and CKM matrices are simply related

$$
\mathcal{U}_{M N S P}=\mathcal{U}_{C K M}^{\dagger} \mathcal{F} \text {. }
$$

In this case, $\mathcal{F}$ must contain two large mixing angles $\eta_{\odot}$ and $\eta_{\oplus}$. As we will discuss in the next section, Eq. (13) then implies that the solar angle $\eta_{\odot}$ experiences a Cabibbo shift $\theta_{\odot} \sim \eta_{\odot} \pm \lambda \cos \eta_{\oplus} \sim \eta_{\odot} \pm \lambda / \sqrt{2}$, and $\theta_{13} \sim \lambda \sin \eta_{\oplus} \sim \lambda / \sqrt{2}$ due to the $\mathcal{O}(\lambda) 1-2$ mixing in $\mathcal{U}_{C K M}$.

The above class of models provides well-motivated examples of leptonic Cabibbo shifts, but it is by no means the only theoretical possibility. In the context of grand unification, the data also can hint that the mixing matrix is initially bimaximal $\left(\eta_{\odot}=\eta_{\oplus}=45^{\circ}\right)$, with the solar angle shifted by a full-strength Cabibbo shift: $\theta_{\odot} \sim \eta_{\odot}-\theta_{c}$ [17, 18, 19, 20]. While these examples can be motivated by flavor theories, one should keep in mind that the data is not yet precise enough to select particular scenarios and the values of the large angles are not known in the $\lambda \rightarrow 0$ limit (if indeed that limit is meaningful for theory). Hence, we will now explore parametrizations of the MNSP matrix which incorporate such leptonic Cabibbo effects purely from a phenomenological standpoint. 


\section{Systematics}

The Wolfenstein parametrization of the CKM matrix is based on the idea that the observed hierarchical quark mixing angles can be understood as powers of the Cabibbo angle $\lambda$, with $\mathcal{U}_{C K M}=1$ in the $\lambda \rightarrow 0$ limit:

$$
\mathcal{U}_{C K M}=1+\mathcal{O}(\lambda) \text {. }
$$

The mixing angles of the quark sector (which have been very precisely determined using direct measurements and unitarity constraints) have the following hierarchical structure [15]:

$$
\mathcal{U}_{C K M}=\left(\begin{array}{ccc}
1-\frac{\lambda^{2}}{2} & \lambda & A \lambda^{3}(\rho-i \eta) \\
-\lambda & 1-\frac{\lambda^{2}}{2} & A \lambda^{2} \\
A \lambda^{3}(1-\rho-i \eta) & -A \lambda^{2} & 1
\end{array}\right)+\mathcal{O}\left(\lambda^{4}\right) .
$$

In the above, $\lambda$ and $A$ are well known $(\lambda=0.22, A \simeq 0.85)$, but $\rho$ and $\eta$ are less precisely determined [21]. The $\mathrm{CP}$-violating phase at lowest order is in the most hierarchically suppressed $1-3$ mixing, but any other choice leads to the same rephasing invariant measure of CP violation. As measured by the JDGW invariant [22, 23], $\mathrm{CP}$ violation in the quark sector is small

$$
J_{C P}^{(C K M)} \simeq A^{2} \lambda^{6} \eta
$$

due to the small mixing angles, not the CP-violating phase, which is the only (possibly) large angle in $\mathcal{U}_{C K M}$.

To seek a similar parametrization for the MNSP matrix, we assume a $\lambda$ expansion of the form

$$
\mathcal{U}_{M N S P}=\mathcal{W}+\mathcal{O}(\lambda)
$$

in which the starting matrix $\mathcal{W}$ has two large angles $\eta_{\oplus}$ and $\eta_{\odot}$, corresponding to the "bare" values of the atmospheric and solar angles, and a vanishing $\mathrm{CHOOZ}$ angle:

$$
\mathcal{W}=\mathcal{R}_{1}\left(\eta_{\oplus}\right) \mathcal{R}_{3}\left(\eta_{\odot}\right) \equiv\left(\begin{array}{ccc}
1 & 0 & 0 \\
0 & \cos \eta_{\oplus} & \sin \eta_{\oplus} \\
0 & -\sin \eta_{\oplus} & \cos \eta_{\oplus}
\end{array}\right)\left(\begin{array}{ccc}
\cos \eta_{\odot} & \sin \eta_{\odot} & 0 \\
-\sin \eta_{\odot} & \cos \eta_{\odot} & 0 \\
0 & 0 & 1
\end{array}\right)
$$

$\mathcal{W}$ is then perturbed by a unitary matrix $\mathcal{V}(\lambda)$. Unlike the case of the quarks, the perturbation matrix does not generically commute with the starting matrix

$$
[\mathcal{W}, \mathcal{V}(\lambda)] \neq 0
$$

resulting in three possible types of Cabibbo shifts (see also [16, 18, 19, 20]):

- Right Cabibbo Shifts:

$$
\mathcal{U}_{M N S P}=\mathcal{W} \mathcal{V}(\lambda)
$$

- Left Cabibbo Shifts:

$$
\mathcal{U}_{M N S P}=\mathcal{V}(\lambda) \mathcal{W}
$$

- Middle Cabibbo Shifts:

$$
\mathcal{U}_{M N S P}=\mathcal{R}_{1}\left(\eta_{\oplus}\right) \mathcal{V}(\lambda) \mathcal{R}_{3}\left(\eta_{\odot}\right)
$$

Given $\eta_{\odot}$ and $\eta_{\oplus}$, one can choose $\mathcal{V}(\lambda)$ to shift the atmospheric and solar angles into the range allowed by the data, and study the resulting implications for $\theta_{13}$ in the three scenarios. A novel feature of our parametrizations is that the JDGW invariant depends not only on the type of Cabibbo shift, but also on how the CP-violating phase is introduced in $\mathcal{V}(\lambda) . J_{C P}^{(M N S P)}$ is typically expected to be much larger than $J_{C P}^{(C K M)}$ due to the larger mixing angles, and can be as large as $\sim \lambda \sin \delta$ (for $\theta_{13} \sim \theta_{c}$ and $\delta \sim \mathcal{O}(1)$ ).

To illustrate these ideas, let us consider models for which Eq. (13) holds, which are left Cabibbo shifts with $\mathcal{F}$ given by Eq. (18) and $\mathcal{V}(\lambda)=\mathcal{U}_{C K M}^{\dagger}$. The shifts in the angles are given to $\mathcal{O}\left(\lambda^{2}\right)$ by

$$
\begin{aligned}
\theta_{\oplus} & =\eta_{\oplus}-\left(A+\frac{1}{4} \sin 2 \eta_{\oplus}\right) \lambda^{2} \\
\theta_{\odot} & =\eta_{\odot}-\cos \eta_{\oplus} \lambda \\
\theta_{13} & =-\lambda \sin \eta_{\oplus} .
\end{aligned}
$$


Using the data to constrain $\mathcal{F}$, assuming the central values $\theta_{\oplus}=45^{\circ}$ and $\theta_{\odot}=32.5^{\circ}$ for simplicity, $\eta_{\oplus} \simeq 48^{\circ}$ (the shift of $\sim 3^{\circ}$ is a typical $\mathcal{O}\left(\lambda^{2}\right)$ correction), $\eta_{\odot} \simeq 41^{\circ}$, and $\theta_{13}=\sin ^{-1}\left(-\lambda \sin \eta_{\oplus}\right) \simeq 9.4^{\circ}$. The JDGW invariant is given by

$$
J_{C P}^{(M N S P)}=\mp \frac{1}{4} \cos \eta_{\oplus} \sin 2 \eta_{\oplus} \sin 2 \eta_{\odot} A \lambda^{3} \eta+\mathcal{O}\left(\lambda^{4}\right),
$$

which is $\sim 10^{-3}$ (setting the CKM parameter $\eta \sim 0.4$ ). As the MNSP matrix has two $\mathcal{O}(1)$ and one $\mathcal{O}(\lambda)$ mixing angles, the effective MNSP phase is suppressed by $\mathcal{O}\left(\lambda^{2}\right)$, even though $J_{C P}^{(M N S P)}$ is larger than $J_{C P}^{(C K M)}$.

\section{Parametrizations}

We now analyze Wolfenstein-like parametrizations of the MNSP matrix which incorporate the different types of leptonic Cabibbo shifts. As Cabibbo shifts are at most $\sim \theta_{c}$ (for $\mathcal{O}(\lambda)$ perturbations), the bare angles $\eta_{\odot}$ and $\eta_{\oplus}$ can e.g. be in the approximate ranges $15^{\circ}<\eta_{\odot}<45^{\circ}, 30^{\circ}<\eta_{\oplus}<60^{\circ}$. One should also keep in mind that the error bars on $\theta_{\oplus}$ and the bound on $\theta_{13}$ are roughly $\mathcal{O}(\lambda)$, while the error bars on $\theta_{\odot}$ are of $\mathcal{O}\left(\lambda^{2}\right)$.

Given $\eta_{\odot}$ and $\eta_{\oplus}$, the perturbation matrix $\mathcal{V}(\lambda)$ must shift the two large angles in the range allowed by the data. Whether the CHOOZ angle is shifted by $\sim \theta_{c}$ or a subleading contribution depends on the details of the $\mathcal{O}(\lambda)$ perturbations in $\mathcal{V}(\lambda)$ and the type of Cabibbo shift, leading to three basic categories:

- Single shift models. These models have only one $\mathcal{O}(\lambda)$ perturbation in $\mathcal{V}(\lambda)$, which can be either in the $1-2$ mixing $\mathcal{V}_{12} \sim \lambda$, the $2-3$ mixing $\mathcal{V}_{23} \sim \lambda$, or the $1-3$ mixing $\mathcal{V}_{13} \sim \lambda$.

- Double shift models. These models have two $\mathcal{O}(\lambda)$ perturbations. There are three possibilities: $\mathcal{V}_{12} \sim$ $\mathcal{V}_{23} \sim \lambda, \mathcal{V}_{12} \sim \mathcal{V}_{13} \sim \lambda$, or $\mathcal{V}_{13} \sim \mathcal{V}_{23} \sim \lambda$

- Triple shift models. These models have $\mathcal{V}_{12} \sim \mathcal{V}_{23} \sim \mathcal{V}_{13} \sim \lambda$.

Focusing for the moment on single shifts, there are several broad classes of parametrizations:

- Cabibbo-shifted atmospheric angle:

In this class of models, the perturbation $\mathcal{V}(\lambda)$ shifts the atmospheric angle by $\sim \theta_{c}$. One possibility is that

$$
\mathcal{V}(\lambda)=\left(\begin{array}{ccc}
1 & 0 & 0 \\
0 & 1 & a \lambda \\
0 & -a \lambda & 1
\end{array}\right)+\mathcal{O}\left(\lambda^{2}\right),
$$

with $a \sim \mathcal{O}(1)$. The solar angle remains unshifted $\left(\theta_{\odot}=\eta_{\odot}\right)$. The shifts in the atmospheric and CHOOZ angles depend on the type of shift scenario. Right shifts

$$
\mathcal{U}_{M N S P}=\mathcal{R}_{1}\left(\eta_{\oplus}\right) \mathcal{R}_{3}\left(\eta_{\odot}\right)\left(\begin{array}{ccc}
1 & 0 & 0 \\
0 & 1 & a \lambda \\
0 & -a \lambda & 1
\end{array}\right)
$$

lead to

$$
\begin{aligned}
\theta_{\oplus} & \simeq \eta_{\oplus}+a \lambda \cos \eta_{\odot}, \\
\theta_{13} & \simeq a \lambda \sin \eta_{\odot},
\end{aligned}
$$

Due to the form of Eq. (28), the size of Cabibbo shifts are $\eta_{\odot}$-dependent. Left shifts

$$
\mathcal{U}_{M N S P}=\left(\begin{array}{ccc}
1 & 0 & 0 \\
0 & 1 & a \lambda \\
0 & -a \lambda & 1
\end{array}\right) \mathcal{R}_{1}\left(\eta_{\oplus}\right) \mathcal{R}_{3}\left(\eta_{\odot}\right)
$$

and middle shifts

$$
\mathcal{U}_{M N S P}=\mathcal{R}_{1}\left(\eta_{\oplus}\right)\left(\begin{array}{ccc}
1 & 0 & 0 \\
0 & 1 & a \lambda \\
0 & -a \lambda & 1
\end{array}\right) \mathcal{R}_{3}\left(\eta_{\odot}\right)
$$


give the same results (to this order in $\lambda$ ):

$$
\begin{aligned}
\theta_{\oplus} & \simeq \eta_{\oplus}+a \lambda, \\
\theta_{13} & \simeq 0 .
\end{aligned}
$$

The atmospheric angle is now shifted by a full-strength Cabibbo effect, and the CHOOZ angle is a higher order effect. If, however,

$$
\mathcal{V}(\lambda)=\left(\begin{array}{ccc}
1 & 0 & a \lambda \\
0 & 1 & 0 \\
-a \lambda & 0 & 1
\end{array}\right)+\mathcal{O}\left(\lambda^{2}\right)
$$

right shifts lead to

$$
\begin{aligned}
\theta_{\oplus} & \simeq \eta_{\oplus}-a \lambda \sin \eta_{\odot} \\
\theta_{13} & \simeq a \lambda \cos \eta_{\odot}
\end{aligned}
$$

while the left and middle shifts leave the atmospheric angle unchanged at this order.

- Cabibbo-shifted solar angle:

In this case, $\mathcal{V}(\lambda)$ shifts the solar angle by $\sim \theta_{c}$. One possibility is that

$$
\mathcal{V}(\lambda)=\left(\begin{array}{ccc}
1 & a \lambda & 0 \\
-a \lambda & 1 & 0 \\
0 & 0 & 1
\end{array}\right)+\mathcal{O}\left(\lambda^{2}\right),
$$

just as in the CKM. The atmospheric angle is unchanged to this order in $\lambda$ for all scenarios. For right shifts

$$
\mathcal{U}_{M N S P}=\mathcal{R}_{1}\left(\eta_{\oplus}\right) \mathcal{R}_{3}\left(\eta_{\odot}\right)\left(\begin{array}{ccc}
1 & a \lambda & 0 \\
-a \lambda & 1 & 0 \\
0 & 0 & 1
\end{array}\right)
$$

and middle shifts

$$
\mathcal{U}_{M N S P}=\mathcal{R}_{1}\left(\eta_{\oplus}\right)\left(\begin{array}{ccc}
1 & a \lambda & 0 \\
-a \lambda & 1 & 0 \\
0 & 0 & 1
\end{array}\right) \mathcal{R}_{3}\left(\eta_{\odot}\right)
$$

the leading order shifts in the solar and $\mathrm{CHOOZ}$ angles are

$$
\begin{aligned}
\theta_{\odot} & \simeq \eta_{\odot}+a \lambda, \\
\theta_{13} & \simeq 0 .
\end{aligned}
$$

In contrast, left shifts

$$
\mathcal{U}_{M N S P}=\left(\begin{array}{ccc}
1 & a \lambda & 0 \\
-a \lambda & 1 & 0 \\
0 & 0 & 1
\end{array}\right) \mathcal{R}_{1}\left(\eta_{\oplus}\right) \mathcal{R}_{3}\left(\eta_{\odot}\right)
$$

yield

$$
\begin{aligned}
\theta_{\odot} & \simeq \eta_{\odot}+a \lambda \cos \eta_{\oplus}, \\
\theta_{13} & \simeq a \lambda \sin \eta_{\oplus} .
\end{aligned}
$$

Left shifts also works if $\mathcal{V}(\lambda)$ is given by Eq. (33), in which case

$$
\begin{aligned}
\theta_{\odot} & \simeq \eta_{\odot}-a \lambda, \\
\theta_{13} & \simeq a \lambda \cos \eta_{\oplus} .
\end{aligned}
$$

Note that here the Cabibbo shifts are sized by $\eta_{\oplus}$-dependent factors. 
- Unshifted solar and atmospheric angles:

In this case, the starting values of the large angles are very close to their central values. There are two possibilities: $\mathcal{V}(\lambda)$ has no entries linear in $\lambda$, or $\mathcal{V}(\lambda)$ is given by Eq. (33), in which case middle Cabibbo shifts

$$
\mathcal{U}_{M N S P}=\mathcal{R}_{1}\left(\eta_{\oplus}\right)\left(\begin{array}{ccc}
1 & 0 & a \lambda \\
0 & 1 & 0 \\
-a \lambda & 0 & 1
\end{array}\right) \mathcal{R}_{3}\left(\eta_{\odot}\right)
$$

lead to $\theta_{13}=a \lambda$ and unshifted large angles.

A similar analysis can be carried out for the double and triple Cabibbo shift scenarios. In analogy with the single shift models, for which either one or two of the three mixing angles receive an $\mathcal{O}(\lambda)$ shift, either two or all three of the mixing angles can receive large Cabibbo shifts in double shift models. Triple shifts lead to $\mathcal{O}(\lambda)$ shifts in all three mixing angles. In Table 1 results are shown for double and triple shifts which are parametrized using one $\mathcal{V}(\lambda)$ matrix (with two or three $\mathcal{O}(\lambda)$ entries) and right, left, or middle shifts.

We do not discuss more complicated ways to introduce $\mathcal{O}(\lambda)$ effects in detail, as they add little to our qualitative conclusions. For example, double and triple shift models can be constructed using two or three single shifts (with $\mathcal{V}(\lambda)$ given by Eq. (27), Eq. (33), or Eq. (35)) and combinations of right, left, and middle shifts. Triple shift models can also incorporate combinations of double and single shifts. These models lead to shifts with similar order of Cabibbo suppression, though their sizes can be different. An illustrative example is a double shift obtained by a left shift with $\mathcal{V}_{12} \sim a \lambda$ and a middle shift with $\mathcal{V}_{13} \sim a^{\prime} \lambda\left(a, a^{\prime} \sim \mathcal{O}(1)\right)$, which yields $\theta_{\odot}=\eta_{\odot}+a \cos \eta_{\oplus} \lambda, \theta_{\oplus}=\eta_{\oplus}$, and $\theta_{13}=\left(a^{\prime}+a \sin \eta_{\oplus}\right) \lambda$.

The various parametrizations can also be classified in terms of the predictions for $\theta_{13}$. Perturbations with $\mathcal{V}_{13} \sim \mathcal{O}(\lambda)$ (such as in Eq. (33)) always lead to $\theta_{13}$ of order $\theta_{c}$. It is also possible to obtain a shift in $\theta_{13}$ of that size through $\mathcal{O}(\lambda)$ entries in $1-2$ mixing with left shifts and $2-3$ mixing with right shifts.

Finally, we note that based on this leading order analysis, many models can be constructed by specifying the bare angles $\eta_{\odot}, \eta_{\oplus}$ and including subleading perturbations. We choose not to do this at this stage, given the wide range of possibilities consistent with current experimental data. Particular parametrizations may emerge as potentially compelling from the standpoint of flavor theory. Improved data, particularly for the $\mathrm{CHOOZ}$ angle, will certainly be invaluable in narrowing the range of possible parametrizations.

\section{CP violation}

Classifying the parametrizations to leading order in $\lambda$ is not sufficient for addressing CP violation, since the JDGW invariants are given by the product of all the entries of $\mathcal{U}_{M N S P}$. Within each of the basic classes of models, examples can be constructed by specifying the details of the subleading perturbations. To illustrate these points, let us consider two representative examples:

- Single shifts with Cabibbo-shifted atmospheric angles, in which $\mathcal{V}(\lambda)$ is given by

$$
\mathcal{V}(\lambda) \sim\left(\begin{array}{ccc}
1 & \mathcal{O}\left(\lambda^{3}\right) & \mathcal{O}\left(\lambda^{2}\right) \\
\mathcal{O}\left(\lambda^{3}\right) & 1 & a \lambda \\
\mathcal{O}\left(\lambda^{2}\right) & -a \lambda & 1
\end{array}\right)
$$

- Double shifts with Cabibbo-shifted atmospheric and solar angles, in which $\mathcal{V}(\lambda)$ is given by

$$
\mathcal{V}(\lambda) \sim\left(\begin{array}{ccc}
1 & a \lambda & \mathcal{O}\left(\lambda^{2}\right) \\
-a \lambda & 1 & a^{\prime} \lambda \\
\mathcal{O}\left(\lambda^{2}\right) & -a^{\prime} \lambda & 1
\end{array}\right)
$$

To include the effects of $\mathrm{CP}$ violation, we allow for a phase $\delta$ of a priori unknown size (though we will assume it is $\mathcal{O}(1)$ ) that can enter either with the dominant or subleading terms of $\mathcal{V}(\lambda)$.

The results, which are presented in Table 2 demonstrate explicitly that the CP-violating invariants depend not only on the details of the subleading perturbations but also on the type of Cabibbo shift, since the mixing 
angles depend on the particular shift scenario. Unlike the quark sector, the leptonic JDGW invariants also depend on whether the CP-violating phase is introduced in the leading or subleading perturbations.

The parametrizations can be classified according to their predictions for the JDGW invariants. As anticipated, the JDGW invariants

$$
J_{C P}^{(M N S P)} \sim\left(\lambda-\lambda^{4}\right) \sin \delta
$$

are much larger than in the quark sector (which is $\sim \lambda^{6}$ ). JDGW invariants of order $\sim \lambda \sin \delta$ are more common for double (and triple) shift scenarios, though single shift models can also predict such large effects.

One generic and novel feature of these parametrizations is that the CP-violating invariants can be much smaller than naive expectations based on the size of the lepton mixing angles, because the effective MNSP phase can be additionally suppressed even for $\delta \sim \mathcal{O}(1)$ (as opposed to the CKM phase, which is $\mathcal{O}(1)$ ). We previously discussed one example, the class of models based on grand unification which satisfy Eq. (13), for which the JDGW invariant is $\mathcal{O}\left(\lambda^{3}\right)$ rather than $\mathcal{O}(\lambda)$, as shown in Eq. (26).

Another illustrative example is the single shift scenario of Eq. (43), with right Cabibbo shifts. In this case, $\theta_{13}$ is predicted to be $\sim \mathcal{O}(\lambda)$ (see Eq. (29)), and hence the JDGW invariant is expected to be $\mathcal{O}(\lambda)$ if the effective MNSP phase is $\mathcal{O}(1)$. As shown in Table 2 this in fact occurs if the $\mathcal{O}(1)$ phase $\delta$ is introduced in

the dominant $2-3$ mixing, for which $J_{C P}^{(M N S P)} \sim \lambda \sin \delta$. However, if $\delta$ is introduced in the most hierarchically suppressed perturbations (as in the quark sector), $\mathcal{V}(\lambda)$ is given by

$$
\mathcal{V}(\lambda)=\left(\begin{array}{ccc}
1 & \left(-\frac{a b}{2}+c e^{-i \delta}\right) \lambda^{3} & b \lambda^{2} \\
-\left(\frac{a b}{2}+c e^{i \delta}\right) \lambda^{3} & 1-\frac{\lambda^{2}}{2} & a \lambda \\
-b \lambda^{2} & -a \lambda & 1-\frac{\lambda^{2}}{2}
\end{array}\right)+\mathcal{O}\left(\lambda^{4}\right)
$$

$(a, b, c \sim \mathcal{O}(1))$. Right shifts predict a suppressed JDGW invariant: $J_{C P}^{(M N S P)} \sim \lambda^{4} \sin \delta$. In this case, a larger CHOOZ angle does not lead to large CP violation because the effective MNSP phase is suppressed by $\mathcal{O}\left(\lambda^{3}\right)$.

Parametrizations which predict a suppressed effective MNSP CP-violating phase abound in Table 2 and appear to be quite generic, reflecting the intriguing possibility that the size of the CHOOZ angle is not necessarily correlated with the magnitude of $\mathrm{CP}$ violation.

\section{Conclusions}

We are beginning to read the new lepton data, but there is much work to do before a credible theory of flavor is proposed. In the meantime, we have found it illustrative to examine the lepton sector through the lens of quark-lepton unification, and investigate parametrizations of the MNSP matrix which include Cabibbosized perturbations. These Wolfenstein-like parametrizations have several novel features, including the generic possibility that the size of the CHOOZ angle is not necessarily correlated with the observability of CP violation.

Our approach emphasizes the need for precision measurements of the MNSP matrix, as the present data is not sufficient to single out a particular parametrization. Should the limit of zero Cabibbo mixing prove to be meaningful for theory, with improved data we may be able to see flavor patterns through the Cabibbo haze.

\section{Acknowledgments}

This work is supported by the United States Department of Energy under grant DE-FG02-97ER41209. A.D. is also supported by the US Department of Energy and Michigan Center for Theoretical Physics.

\section{References}

[1] Z. Maki, M. Nakagawa and S. Sakata, Prog. Theor. Phys. 28, 870 (1962).

[2] B. Pontecorvo, Sov. Phys. JETP 6, 429 (1957) [Zh. Eksp. Teor. Fiz. 33, 549 (1957)].

[3] The SNO Collaboration, S.N. Ahmed et al., Phys.Rev.Lett. 92 (2004) 181301. 
[4] The Super-Kamiokande Collaborations, S. Fukuda et al., Phys.Lett.B 539 (2002) 179.

[5] The Super-Kamiokande Collaborations, S. Fukuda et al., Phys.Rev.Lett. 81 (1998) 1562; ibid 85 (2000) 3999.

[6] The KamLAND Collaboration, K. Eguchi et al., Phys.Rev.Lett. 90 (2003) 021802.

[7] The CHOOZ Collaboration, M. Apollonio et al., Phys.Lett. B 338 (1998) 383; ibid 420 (1998) 397; ibid 466 (1999) 415; the Palo Verde collaboration, F. Boehm et al., Phys. Rev. Lett. 84, 3764 (2000) hep-ex/9912050. For improved treatments leading to significant relaxation of the upper bound see M. Apollonio et al., Eur.Phys.J. C27 (2003) 331.

[8] The K2K Collaboration, M.H. Ahn et al., Phys.Rev.Lett. 90 (2003) 041801.

[9] M. Maltoni et al., New J.Phys. 6 (2004) 122; M. C. Gonzalez-Garcia and M. Maltoni, hep-ph/0406056 S. Goswami, A. Bandyopadhyay and S. Choubey, hep-ph/0409224.

[10] M. Freund, P. Huber and M. Lindner, Nucl. Phys. B 615, 331 (2001) hep-ph/0105071.

P. Huber, M. Lindner and W. Winter, Nucl.Phys. B 645 (2002) 3; M. Apollonio et al., hep-ph/0210192 P. Huber et al., Nucl.Phys. B 665 (2003) 487; S. Pakvasa and J.W.F. Valle, Proc. Indian Natl. Sci. Acad. 70A, 189 (2004) hep-ph/0301061; V. Barger, D. Marfatia and K. Whisnant, hep-ph/0308123 and references therein; "White Paper Report on Using Nuclear Reactors to Search for a Value of $\theta_{13}$ ", K. Anderson et al., January, 2004, document available at http://www.hep.anl.gov/minos/reactor13/white.html

[11] T. Kajita, talk given at NOON-2004, Tokyo, Feb. 2004.

[12] J. C. Pati and A. Salam, Phys. Rev. D 10, 275 (1974).

[13] M. Gell-Mann, P. Ramond, and R. Slansky in Sanibel Talk, CALT-68-709, Feb 1979 retropreprinted as hep-ph/9809459, and in Supergravity (North Holland, Amsterdam 1979). T. Yanagida, in Proceedings of the Workshop on Unified Theory and Baryon Number of the Universe, KEK, Japan, Feb 1979. P. Minkowski, Phys. Lett. B 67, 421 (1977).

[14] A. Datta, F. S. Ling and P. Ramond, Nucl. Phys. B 671, 383 (2003) hep-ph/0306002.

[15] L. Wolfenstein, Phys. Rev. Lett. 51, 1945 (1983).

[16] P. Ramond, hep-ph/0401001, hep-ph/0405176 Nucl. Phys. Proc. Suppl. 137, 317 (2004) hep-ph/0411009; hep-ph/0411010

[17] H. Minakata and A. Y. Smirnov, Phys. Rev. D 70, 073009 (2004) hep-ph/0405088; A. Y. Smirnov, hep-ph/0402264 M. Raidal, Phys. Rev. Lett. 93, 161801 (2004); P. H. Frampton and R. N. Mohapatra, JHEP 0501, 025 (2005); S. T. Petcov and W. Rodejohann, hep-ph/0409135.

[18] C. Giunti and M. Tanimoto, Phys. Rev. D 66, 053013 (2002) hep-ph/0207096.

[19] P. H. Frampton, S. T. Petcov and W. Rodejohann, Nucl. Phys. B 687, 31 (2004) hep-ph/0401206; W. Rodejohann, Phys. Rev. D 69, 033005 (2004);

[20] K. Cheung, S. K. Kang, C. S. Kim and J. Lee, hep-ph/0503122

[21] S. Eidelman et al., Phys. Lett. B 592, 1 (2004).

[22] C. Jarlskog, Phys. Rev. Lett. 55, 1039 (1985).

[23] I. Dunietz, O. W. Greenberg and D. d. Wu, Phys. Rev. Lett. 55, 2935 (1985). 


\begin{tabular}{|c|c|c|c|}
\hline Double Shifts & Right & Left & Middle \\
\hline $\begin{array}{l}\mathcal{V}_{12} \sim a \lambda \\
\mathcal{V}_{23} \sim a^{\prime} \lambda\end{array}$ & $\begin{array}{c}\theta_{\odot}=\eta_{\odot}+a \lambda \\
\theta_{\oplus}=\eta_{\oplus}+a^{\prime} \lambda \cos \eta_{\odot} \\
\theta_{13}=a^{\prime} \sin \eta_{\odot} \lambda\end{array}$ & $\begin{array}{c}\theta_{\odot}=\eta_{\odot}+a \cos \eta_{\oplus} \lambda \\
\theta_{\oplus}=\eta_{\oplus}+a^{\prime} \lambda \\
\theta_{13}=a \sin \eta_{\oplus} \lambda\end{array}$ & $\begin{array}{c}\theta_{\odot}=\eta_{\odot}+a \lambda \\
\theta_{\oplus}=\eta_{\oplus}+a^{\prime} \lambda \\
\theta_{13}=0\end{array}$ \\
\hline $\begin{array}{l}\mathcal{V}_{12} \sim a \lambda \\
\mathcal{V}_{13} \sim a^{\prime} \lambda\end{array}$ & $\begin{array}{c}\theta_{\odot}=\eta_{\odot}+a \lambda \\
\theta_{\oplus}=\eta_{\oplus}-a^{\prime} \lambda \cos \eta_{\odot} \\
\theta_{13}=a^{\prime} \cos \eta_{\odot} \lambda\end{array}$ & $\begin{array}{c}\theta_{\odot}=\eta_{\odot}+\left(a \cos \eta_{\oplus}-a^{\prime} \sin \eta_{\oplus}\right) \lambda \\
\theta_{\oplus}=\eta_{\oplus} \\
\theta_{13}=\left(a \sin \eta_{\oplus}+a^{\prime} \cos \eta_{\oplus}\right) \lambda\end{array}$ & $\begin{array}{c}\theta_{\odot}=\eta_{\odot}+a \lambda \\
\theta_{\oplus}=\eta_{\oplus} \\
\theta_{13}=a^{\prime} \lambda\end{array}$ \\
\hline $\begin{array}{l}\mathcal{V}_{13} \sim a \lambda \\
\mathcal{V}_{23} \sim a^{\prime} \lambda\end{array}$ & $\begin{array}{c}\theta_{\odot}=\eta_{\odot} \\
\theta_{\oplus}=\eta_{\oplus}+\left(a^{\prime} \cos \eta_{\odot}-a \sin \eta_{\odot}\right) \lambda \\
\theta_{13}=\left(a \cos \eta_{\odot}+a^{\prime} \sin \eta_{\odot}\right) \lambda\end{array}$ & $\begin{array}{c}\theta_{\odot}=\eta_{\odot}-a \sin \eta_{\oplus} \lambda \\
\theta_{\oplus}=\eta_{\oplus}+a^{\prime} \lambda \\
\theta_{13}=a \cos \eta_{\oplus} \lambda\end{array}$ & $\begin{array}{c}\theta_{\odot}=\eta_{\odot} \\
\theta_{\oplus}=\eta_{\oplus}+a^{\prime} \lambda \\
\theta_{13}=a \lambda\end{array}$ \\
\hline Triple Shifts & Right & Left & Middle \\
\hline $\begin{aligned} \mathcal{V}_{12} & \sim a \lambda \\
\mathcal{V}_{23} & \sim a^{\prime} \lambda \\
\mathcal{V}_{13} & \sim a^{\prime \prime} \lambda\end{aligned}$ & $\begin{array}{c}\theta_{\odot}=\eta_{\odot}+a \lambda \\
\theta_{\oplus}=\eta_{\oplus}+\left(a^{\prime} \cos \eta_{\odot}-a^{\prime \prime} \sin \eta_{\odot}\right) \lambda \\
\theta_{13}=\left(a^{\prime \prime} \cos \eta_{\odot}-a^{\prime} \sin \eta_{\odot}\right) \lambda\end{array}$ & $\begin{array}{c}\theta_{\odot}=\eta_{\odot}+\left(a \cos \eta_{\oplus}-a^{\prime \prime} \sin \eta_{\oplus}\right) \lambda \\
\theta_{\oplus}=\eta_{\oplus}+a^{\prime} \lambda \\
\theta_{13}=\left(a^{\prime \prime} \cos \eta_{\oplus}+a \sin \eta_{\oplus}\right) \lambda\end{array}$ & $\begin{array}{c}\theta_{\odot}=\eta_{\odot}+a \lambda \\
\theta_{\oplus}=\eta_{\oplus}+a^{\prime} \lambda \\
\theta_{13}=a^{\prime \prime} \lambda\end{array}$ \\
\hline
\end{tabular}

Table 1: Leading order shifts in the MNSP mixing angles for double and triple shifts $\left(a, a^{\prime}, a^{\prime \prime} \sim \mathcal{O}(1)\right)$.

Table 2: JDGW invariants for a representative single shift scenario with $\mathcal{V}(\lambda)$ given by Eq. (43) and double shift scenario with $\mathcal{V}(\lambda)$ given by Eq. (44). In column 1, the label denotes the placement of the phase in the subblock of the perturbing matrix. $a\left(a^{\prime}\right), b$, and $c$ are $\mathcal{O}(1)$ parameters associated with the $\mathcal{O}(\lambda), \mathcal{O}\left(\lambda^{2}\right)$, and $\mathcal{O}\left(\lambda^{3}\right)$ perturbations (see e.g. Eq. (46)).

\begin{tabular}{|c|c|c|c|}
\hline Single & Right & Left & Middle \\
\hline 12 & $\frac{a b}{16}\left(\sin 3 \eta_{\odot}-7 \sin \eta_{\odot}\right) \sin 2 \eta_{\oplus} \lambda^{4} \sin \delta$ & $-\frac{c}{4} \sin 2 \eta_{\odot} \sin \eta_{\oplus} \sin 2 \eta_{\oplus} \lambda^{3} \sin \delta$ & $\frac{a c}{8} \sin 2 \eta_{\odot} \sin 2 \eta_{\oplus} \lambda^{4} \sin \delta$ \\
13 & $\frac{b}{4} \cos \eta_{\odot} \sin 2 \eta_{\odot} \sin 2 \eta_{\oplus} \lambda^{2} \sin \delta$ \\
23 & $\frac{a}{4} \sin \eta_{\odot} \sin 2 \eta_{\odot} \sin 2 \eta_{\oplus} \lambda \sin \delta$ & $-\frac{a b}{16}\left(7 \cos \eta_{\oplus}+\cos 3 \eta_{\oplus}\right) \sin 2 \eta_{\odot} \lambda^{3} \sin \delta$ & $\begin{array}{c}\frac{b}{4} \sin 2 \eta_{\odot} \sin 2 \eta_{\oplus} \lambda^{2} \sin \delta \\
-\frac{a b}{2} \cos 2 \eta_{\oplus} \sin 2 \eta_{\odot} \lambda^{3} \sin \delta\end{array}$ \\
\hline Double & Right & Left & Middle \\
\hline 12 & $\frac{a a^{\prime}}{16}\left(\sin 3 \eta_{\odot}-7 \sin \eta_{\odot}\right) \sin 2 \eta_{\oplus} \lambda^{2} \sin \delta$ & $\frac{a}{4} \sin 2 \eta_{\odot} \sin \eta_{\oplus} \sin 2 \eta_{\oplus} \lambda \sin \delta$ & $\frac{a a^{\prime}}{8} \sin 2 \eta_{\odot} \sin 2 \eta_{\oplus} \lambda^{2} \sin \delta$ \\
13 & $\frac{b}{4} \cos \eta_{\odot} \sin 2 \eta_{\odot} \sin 2 \eta_{\oplus} \lambda^{2} \sin \delta$ & $\frac{b}{4} \cos \eta_{\oplus} \sin 2 \eta_{\odot} \sin 2 \eta_{\oplus} \lambda^{2} \sin \delta$ & $\frac{b}{4} \sin 2 \eta_{\odot} \sin 2 \eta_{\oplus} \lambda^{2} \sin \delta$ \\
23 & $\frac{a^{\prime} \sin \eta_{\odot} \sin 2 \eta_{\odot} \sin 2 \eta_{\oplus} \lambda \sin \delta}{4}$ & $\frac{a a^{\prime}}{16}\left(\sin 3 \eta_{\oplus}-7 \sin \eta_{\oplus}\right) \sin 2 \eta_{\odot} \lambda^{2} \sin \delta$ & $\frac{a a^{\prime}}{8} \sin 2 \eta_{\oplus} \sin 2 \eta_{\odot} \lambda^{2} \sin \delta$ \\
\hline
\end{tabular}

